\title{
Preparation of mesophase pitch by aromatics-rich distillate of naphthenic vacuum gas oil
}

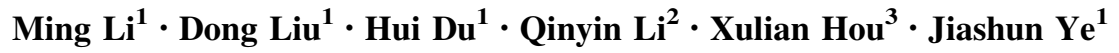

Received: 5 April 2015/Accepted: 29 June 2015/Published online: 9 July 2015

(C) The Author(s) 2015. This article is published with open access at Springerlink.com

\begin{abstract}
Two aromatics-rich distillates R1 and R2 with different properties from naphthenic base vacuum gas oil were used for preparing mesophase pitch through highpressure thermal treatment. ${ }^{1} \mathrm{H}-\mathrm{NMR}$, FT-IR and VPO were employed to characterize the structural parameters of the raw materials. The products' optical texture and molecular structure were analyzed by polarized light optical microscopy, ${ }^{1} \mathrm{H}-\mathrm{NMR}$, FT-IR and XRD. The effect of raw materials' structure on the formation of mesophase pitch was discussed. The results showed that the structure of the raw material had an important effect on the formation of mesophase pitch. The raw material R2 with higher aromaticity, more naphthenic structure and less alkyl side chains was easy to form mesophase pitch with large-domains optical texture, lower softening point and more ordered crystal structure.
\end{abstract}

Keywords Mesophase pitch - Naphthenic vacuum gas oil · Optical texture - Molecular structure . Crystal structure

Dong Liu

ldongupc@vip.sina.com

1 State Key Laboratory of Heavy Oil Processing, China University of Petroleum, Qingdao 266580, China

2 College of Science, China University of Petroleum, Qingdao 266580, China

3 China Petroleum Engineering Co., Ltd., Beijing Company, Beijing 100085, China

\section{Introduction}

Mesophase pitch prepared by bitumen, heavy oil, coal tar and other raw materials through thermal condensation reaction is recognized as the precursor of carbon fiber, needle coke, carbon electrode material, foam materials and other raw materials with excellent advanced features [1-4]. It has been well established that the molecular weight and alkyl groups of a raw material are primary factors controlling the solubility and anisotropic of resultant carbons [5-7]. In particular, the high concentration of alkyl group in pitch seemed to increase the solubility of the mesophase, thereby the optical structure of mesophase pitch was improved [8].

Mochida et al. [9-11] reported that hydrogenation and reductive alkylation methods were effective methods to convert the quinolone-insoluble fractions into graphitizable carbon. After studying the preparation of naphthalene mesophase pitch and methyl naphthalene mesophase pitch, Korai and Mochida [12] suggested that the methyl in mesophase molecule was the main factor to the stacking of mesogen molecules. It has been reported that a nematic liquid crystal which was a typical mesophase material was produced by heavy aromatic hydrocarbons during the heat treatment [13, 14]. The heavy fraction of light diesel oil, derived from petroleum naphtha catalytic cracking, was chosen as the additives in co-carbonization of medium coal tar pitch to modify the carbonization property. The results showed that the mesophase pitch optical structure changed from the original mosaic into a large-flow domain $[15,16]$. The composition of aromatics-rich fraction in catalytic slurry (FCCRF) and the carbonization behavior have been studied [17]. The result showed that the carbonization behavior was influenced by the composition of feedstock substantially, so the selection of raw materials was very 
important. To be specific, a mesophase pitch with an orderly disk-like structure could be formed from a feedstock with feasible composition. But the influences of the alkyl side chains and naphthenic groups on the optical and molecular structures of mesophase pitch have not been studied systematically so far as we know.

In this work, two batches of aromatics-rich distillates R1 and R2 with different properties were derived from naphthenic base vacuum gas oil by furfural extraction, then mesophase pitches were prepared by thermal treatment using R1 and R2 as raw materials, respectively, namely R1-MP and R2-MP. The influences of the raw materials on the properties of mesophase pitch were studied, and the carbonization mechanism was discussed preliminarily.

\section{Experimental}

\section{Materials}

Two aromatics-rich distillates R1 and R2 with different properties are derived from naphthenic vacuum gas oil.

The characteristics of R1 and R2 are listed in Table 1. From Table 1, the two oils contain no asphaltene. Compared with R1, R2 contains more aromatics but less saturates and resin. The relative molecular mass of $\mathrm{R} 2$ is higher than $\mathrm{R} 1$, while the $\mathrm{n}(\mathrm{H}): \mathrm{n}(\mathrm{C})$ was opposite. It indicates that the condensation degree of R2 was higher than that of R1.

\section{Experimental methods}

The raw material was placed in the 100-ml high-pressure autoclave and heated in an electric furnace to $440-450{ }^{\circ} \mathrm{C}$ with a rate of less than $3{ }^{\circ} \mathrm{C} / \mathrm{min}$ after purging the reaction system by nitrogen for three times. During the experiment, the pressure was maintained at about $4 \mathrm{MPa}$. After the pyrogenation process at $440-445^{\circ} \mathrm{C}$, the reaction was carried out under $4 \mathrm{MPa}$ for $2-12 \mathrm{~h}$.

\section{Analysis}

The elemental compositions of samples were obtained from Germany Chnos company Vario EL II elemental analyzer. The relative molecular mass of the raw materials were obtained from German Knauer Company Vapour Pressure Osmometer K-700 molecular weight apparatus. The microstructures were observed and photographed by BX51-P polarizing microscope with camera (made in Germany). FT-IR spectra were obtained on US Digilet Company FT S215 Fourier transform-infrared spectroscopy. Solid samples were dissolved in benzene first and smeared on $\mathrm{NaCl}$ single-crystal flakes uniformly, then characterized after the solution was volatilized. ${ }^{1} \mathrm{H}-\mathrm{NMR}$ spectra were obtained from US Varian Company Unity$200 \mathrm{MHz}$ FT NMR spectrometer. Tetramethylsilane (TMS) was added as an internal standard to samples that were dissolved in $\mathrm{CDCl}_{3}$. The operating frequency is $80 \mathrm{MHz}$, and the scanning width is $2 \mathrm{kHz}$. The group composition of mesophase pitch was analyzed according to SH/T 0509-92.

\section{Results and discussion}

\section{FT-IR analysis}

The FT-IR spectra of R1 and R2 are shown in Fig. 1. It can be observed from Fig. 1 that two kinds of raw materials present strong absorption peaks at 1460, 2853, 2923, 1380 and $2960 \mathrm{~cm}^{-1}$, indicating that the raw materials contain many alkyl side chains. The molar ratio of $-\mathrm{CH}_{2}-(\mathrm{ab}-$ sorption peaks at $1460 \mathrm{~cm}^{-1}$ ) to $-\mathrm{CH}_{3}$ (absorption peaks at $1460 \mathrm{~cm}^{-1}$ ) can be evaluated according to the following formula [18].

$r=3.07 \frac{A_{1460}}{A_{1380}}-3.72$

In the formula, $A_{1460}$ and $A_{1380}$ represent absorption peak intensities at 1460 and $1380 \mathrm{~cm}^{-1}$. The results showed that the ratios of R1 and R2 were 2.01 and 0.76 , suggesting that the alkyl side chain of R2 was shorter than that of R1. The aromaticity $\left(f_{\mathrm{a}}\right)$ of $\mathrm{R} 1$ and $\mathrm{R} 2$ was calculated according to the following formula [18].

$f_{\mathrm{a}}=0.574 P+0.024$

In the formula, $P$ is defined as $A_{1600} /\left(A_{1600}+0.16\right.$ $A_{1460}+0.23 A_{1330}$ ), and $A_{1660}$ represents absorption peak

Table 1 Basic properties of feedstocks

\begin{tabular}{|c|c|c|c|c|c|c|c|c|}
\hline \multirow[t]{2}{*}{ Sample } & \multicolumn{4}{|l|}{ wt $\%$} & \multicolumn{2}{|l|}{ wt $\%$} & \multirow[t]{2}{*}{$\mathrm{n}(\mathrm{H}): \mathrm{n}(\mathrm{C})$} & \multirow[t]{2}{*}{ M } \\
\hline & Saturates & Aromatics & Resin & Asphaltene & $\mathrm{C}$ & $\mathrm{H}$ & & \\
\hline R1 & 22.26 & 62.94 & 13.69 & 0 & 87.93 & 9.94 & 1.36 & 383.20 \\
\hline R2 & 16.51 & 71.67 & 10.87 & 0 & 90.12 & 7.49 & 1.00 & 526.51 \\
\hline
\end{tabular}

$M$ relative molecular mass 


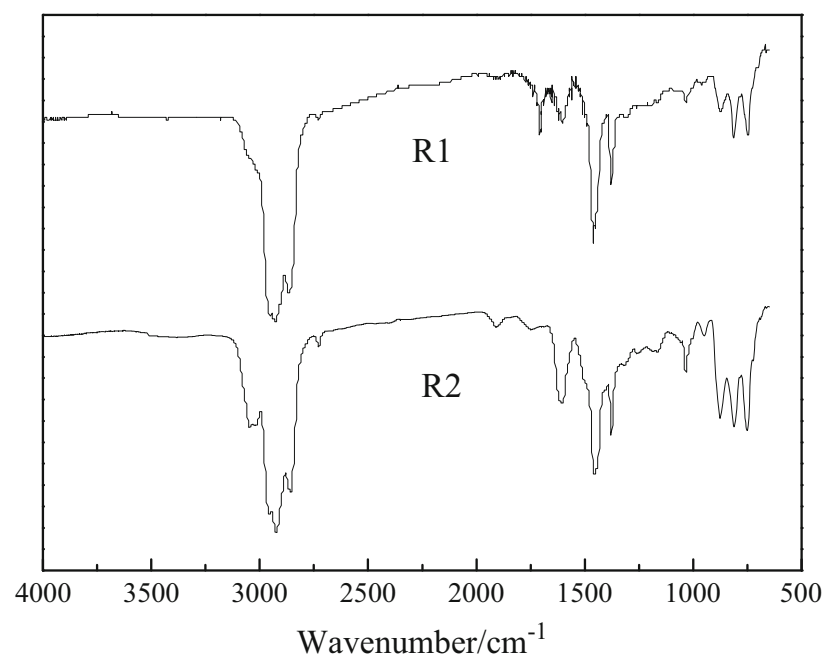

Fig. 1 FT-IR spectra of raw materials

intensity at $1660 \mathrm{~cm}^{-1}$. The aromaticity $\left(f_{\mathrm{a}}\right)$ of $\mathrm{R} 1$ and $\mathrm{R} 2$ was 0.37 and 0.62 , implying that the aromaticity of R2 was higher than that of R1.

The FT-IR spectra of R1-MP-8h (mesophase pitch prepared from R1 for $8 \mathrm{~h}$ ) and R2-MP-8h (mesophase pitch prepared from R2 for $8 \mathrm{~h}$ ) are shown in Fig. 2. The aliphatic hydrocarbon absorption intensity $\left(3000-2845 \mathrm{~cm}^{-1}\right)$ of R1-MP-8h and R2-MP-8h increased while condensed aromatics absorption intensity $\left(900-650 \mathrm{~cm}^{-1}\right.$, $1650-1450 \mathrm{~cm}^{-1}$ ) decreased as compared to the raw materials. It suggested a fracture process of long aliphatic side chains and a polycondensation process of molecules. The aromatic indexes $\left(I_{\mathrm{ar}}\right)$ of R1-MP-8h and R2-MP-8h calculated by the following formula [18] were 0.76 and 0.69 , indicating that R2-MP-8h contained more aliphatic groups than R1-MP-8h.

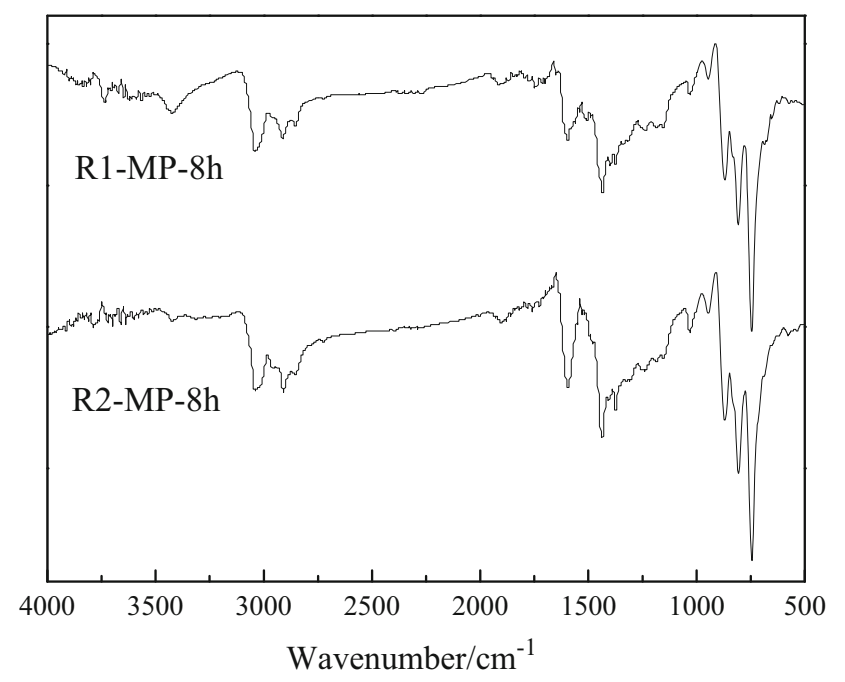

Fig. 2 FT-IR spectra of the mesophase pitches
$I_{\mathrm{ar}}=\frac{A b_{3040}}{A b_{3040}+A b_{2920}}$

The $880 / 1600 \mathrm{~cm}^{-1}$ peak intensity ratio (isolated aromatic $\mathrm{C}-\mathrm{H} /$ aromatic $\mathrm{C}=\mathrm{C}$ ) were used to reflect the condensation degrees of polycyclic aromatic hydrocarbons (PAH) [18], and the PAH of R1-MP-8h and R2-MP-8h were 2.71 and 1.05 , demonstrating that the condensation degrees of R2-MP-8h were lower than that of R1-MP-8h. The result was consistent with the change of aromatic indexes.

\section{${ }^{1}$ H-NMR analysis}

The ${ }^{1}$ H-NMR spectra of R1 and R2 are shown in Fig. 3 and the analysis results are shown in Table 2 . It revealed that the $\mathrm{H}_{\alpha}$ and $\mathrm{H}_{\gamma}$ contents of R2 were higher than that of R1, while the $\mathrm{H}_{\mathrm{ar}}$ content was opposite, indicating that $\mathrm{R} 1$ contained more alkyl side chains than R2. The alkyl side chains could improve the reaction rate to a certain extent. But if the raw material contained excessive amounts of alkyl side chains, it would produce more free radicals [19, 20], which make the polycondensation reaction very fast and hinder formation of a mesophase phase with low softening point [9-13]. The $H_{n}$ contents of $\mathrm{R} 1$ and $\mathrm{R} 2$ were 22.32 and 35.17 , illustrating that $\mathrm{R} 2$ contained more naphthenic groups. The structural parameters of the raw materials shown in Table 2 were calculated using the improved Brown-Ladner method [18]. The aromaticity $\left(f_{\mathrm{a}}\right)$ of R1 and R2 was 0.39 and 0.66 , which was consistent with the FT-IR analysis results.

The pyridine-soluble fractions of R1-MP-8h and R2MP-8h (R1-MP-8h-PS and R2-MP-8h-PS) were analyzed by ${ }^{1} \mathrm{H}-\mathrm{NMR}$. The spectra of R1-MP-8h-PS and R2-MP-8hPS are shown in Fig. 4 and the distribution of the hydrogen atoms is tabulated in Table 3 .

As shown in Table 3, the $\mathrm{H}_{\alpha}$ and $\mathrm{H}_{\gamma}$ contents of R2-MP8h-PS were higher than that of R1-MP-8h-PS, while the $\mathrm{H}_{\mathrm{ar}}$ content was opposite, indicating that R2-MP-8h-PS contained more alkyl side chains than R1-MP-8h-PS. The $\mathrm{H}_{\mathbf{n}}$ content of R1-MP-8h-PS and R2-MP-8h-PS was 0.98 and 3.72, implying that R2-MP-8h-PS contained more naphthenic groups. The absorption peaks of $-\mathrm{CH}_{2}-$ at 3.3-4.2 indicated that the aromatic layers were connected with each other by $-\mathrm{CH}_{2}$ bonds [21]. The contents of $\mathrm{H}_{\beta}$ and $\mathrm{H}_{\gamma}$ in mesophase pitches decreased markedly as compared with the raw materials, illustrating that the pyrolysis process was mainly to remove $\mathrm{H}_{\beta}$ and $\mathrm{H}_{\gamma}$, and their removal resulted in the generation of aromatic intermediates which were stable aromatic radicals $[8,15]$. The aromaticity $\left(f_{\mathrm{a}}\right)$ of R1-MP8h-PS and R2-MP-8h-PS calculated by improved n-d-M method [18] was 0.76 and 0.65 , implying that R2-MP-8hPS contained more aliphatic groups, which was consistent to the results of FT-IR analysis.

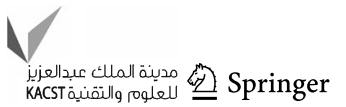




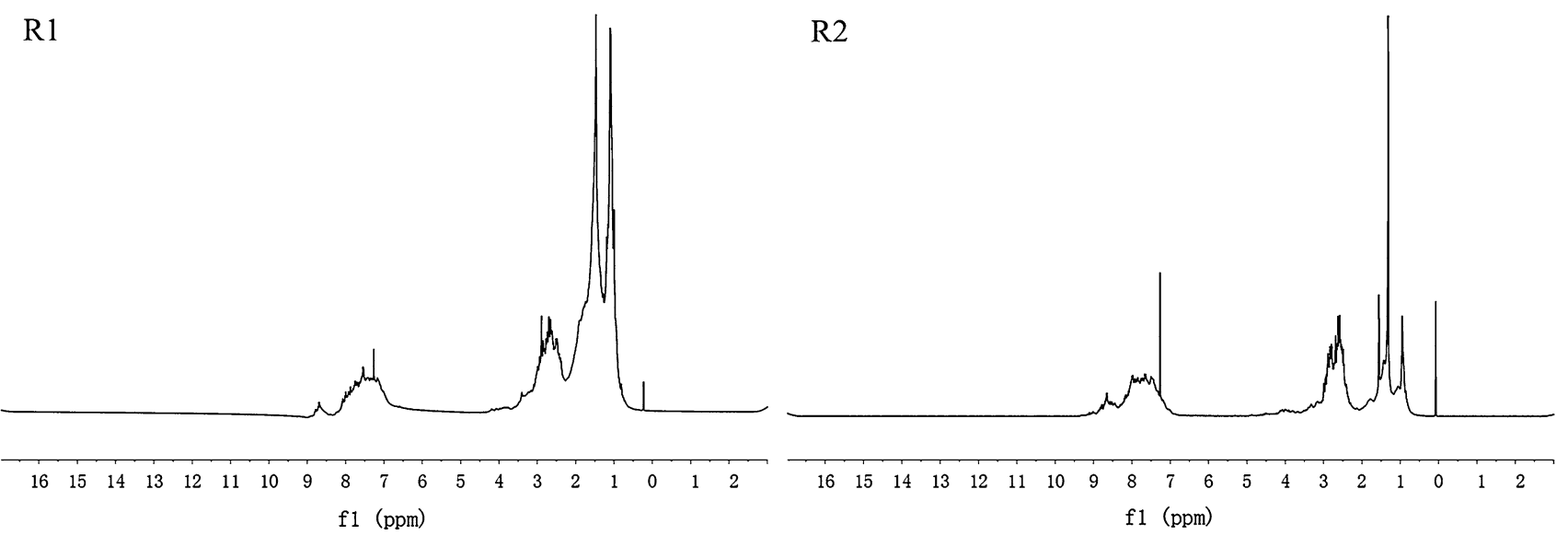

Fig. $3{ }^{1} \mathrm{H}-\mathrm{NMR}$ spectrum of the raw materials

Table 2 Structural parameters of the raw materials

\begin{tabular}{lllllll}
\hline Sample & $\mathrm{H}_{\mathrm{ar}}$ & $\mathrm{H}_{\alpha}$ & $\mathrm{H}_{\beta}$ & $\mathrm{H}_{\gamma}$ & $\mathrm{H}_{\mathrm{n}}$ & \\
\hline $\mathrm{R} 1$ & 12.74 & 21.39 & 17.53 & 26.02 & 22.32 & 0.39 \\
$\mathrm{R} 2$ & 32.25 & 14.57 & 6.49 & 11.52 & 35.17 & 0.66 \\
\hline
\end{tabular}

$\mathrm{H}_{\mathrm{ar}}$, aromatic hydrogens; $\mathrm{H}_{\alpha}$, aliphatic hydrogens in methyl or methylene groups in $\alpha$-position to an aromatic ring $(3.3-2.0 \mathrm{ppm}) ; \mathrm{H}_{\mathrm{n}}$, naphthenic hydrogen (2.0-1.4 ppm); $\mathrm{H}_{\beta}$, aliphatic hydrogens in methyl or methylene groups in $\beta$-position to an aromatic ring (1.4-1.0 ppm); $\mathrm{H}_{\gamma}$, aliphatic hydrogens in methyl or methylene groups in $\gamma$-position to an aromatic ring (1.0-0.5 ppm); $f_{\text {a }}$, aromaticity

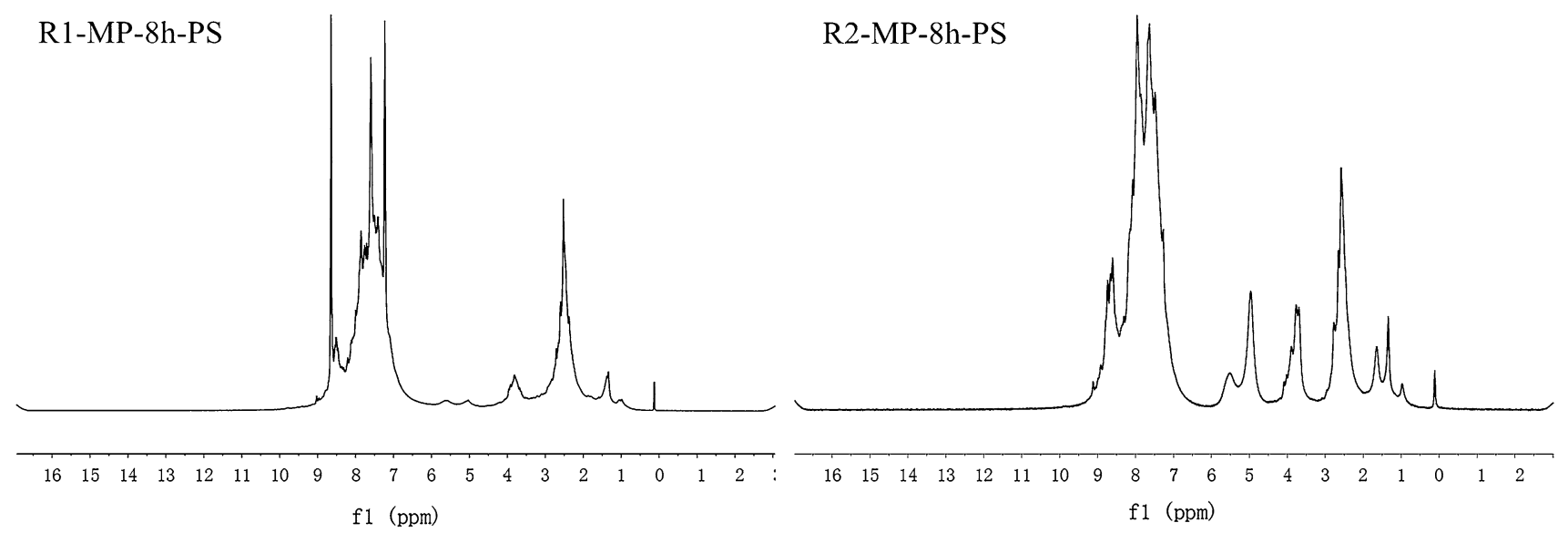

Fig. $4{ }^{1} \mathrm{H}$-NMR spectra of MP-PS

Table 3 Hydrogen atoms distribution of MP-PS

\begin{tabular}{|c|c|c|c|c|c|}
\hline Samples & $\mathrm{H}_{\mathrm{ar}}(\%)$ & $\mathrm{H}_{\alpha}(\%)$ & $\mathrm{H}_{\beta}(\%)$ & $\mathrm{H}_{\gamma}(\%)$ & $\mathrm{H}_{\mathrm{n}}(\%)$ \\
\hline R1-MP-8h-PS & 87.95 & 5.84 & 3.81 & 1.42 & 0.98 \\
\hline R2-MP-8h-PS & 72.71 & 16.29 & 4.92 & 2.36 & 3.72 \\
\hline
\end{tabular}




\section{Optical textures analysis}

The optical micrographs of R1-MP and R2-MP are shown in Figs. 5 and 6. As shown in Fig. 5, a number of small spheres and a few of ball aggregates were formed after $4 \mathrm{~h}$. A mesophase pitch with fine mosaic structure was generated after $6 \mathrm{~h}$. With the development of the reaction, it was observed that the mesophase pitch with medium domain structure (diameter in $100 \mu \mathrm{m}-200 \mu$ mon average) was formed after $8 \mathrm{~h}$. Moreover, the mesophase content of R1MP- $8 \mathrm{~h}$ was $87 \%$ and the softening point was $289^{\circ} \mathrm{C}$. When the carbonization time was too long (over $12 \mathrm{~h}$ ), a mesophase pitch with fine mosaic structure was formed, because the over-carbonization destroyed the crystal structure. From the optical micrographs of R2-MP shown in Fig. 6, we found that the changing tendency of R2-MP was consistent with that of R1-MP. Due to the high condensation degree of $\mathrm{R} 2$, it just needed $4 \mathrm{~h}$ to obtain a mesophase pitch with coarse mosaic structure. A mesophase pitch with large-domain structure (the diameter were around $200 \mu \mathrm{m}$ ) appeared after $6 \mathrm{~h}$. This large-domain structure was retained after coking for $10 \mathrm{~h}$. Moreover, the mesophase content of R2-MP-8h (a mesophase pitch prepared using R2 as raw material by thermal treatment after $8 \mathrm{~h}$ ) was $95 \%$ and the softening point was $243{ }^{\circ} \mathrm{C}$. However, a mesophase pitch with coarse mosaic structure was formed because of the overreaction.

In summary, R1 leads to a mesophase pitch with medium domain structure after $8 \mathrm{~h}$, while the mesophase pitch that produced from R2 had large-domain structure after $6 \mathrm{~h}$. The results show that R2 can be generated into highquality mesophase pitch under certain conditions through carbonization alone, while R1 cannot.

\section{Yield analysis}

Four fractions $n$-heptane-soluble fraction (HS), $n$-heptaneinsoluble/toluene-soluble fraction (HI-TS), toluene-insoluble/pyridine-soluble fraction (TI-PS) and pyridine-insoluble fraction (PI) of the product are obtained by extracting with heptane, toluene and pyridine. The yields of fractions refer to the mass percentages of fractions. The yield-time curves of HS, HI-TS, TI-PS and PI in mesophase pitches are shown in Fig. 7. The changing tendencies of fraction yields in R1-MP were similar to those of R2-MP. The HS yields decreased and the PI yields increased with the proceeding of carbonizing treatment, while the HI-TS yields and TI-PS yields increased at first and then decreased. The reason was that HS-TI and TI-PS were the intermediate products and its yields changed depending on its production rate and consumption rate $[22,23]$. The yield of R2MP was lower than that of R1-MP at the end of the reaction.

During the process of thermal treatment, the HI-TS and TI-PS yields of R2-MP changed more slowly than that of R1-MP. The reason may be that the hydrogen transfer reaction took place owing to the naphthenic groups which could produce macromolecular free
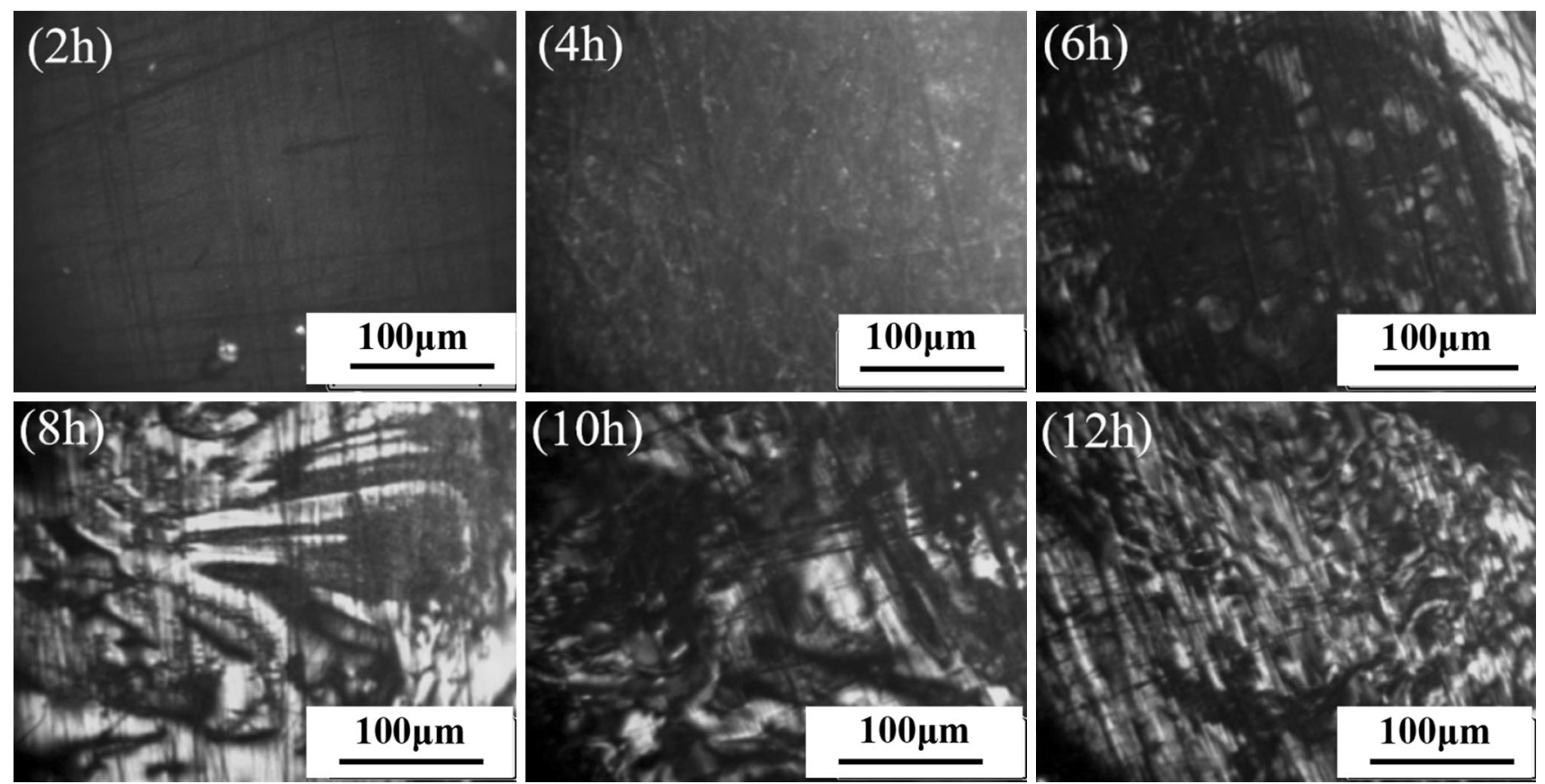

Fig. 5 Optical micrographs of R1-MP

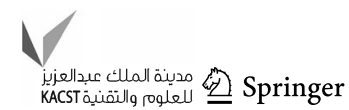



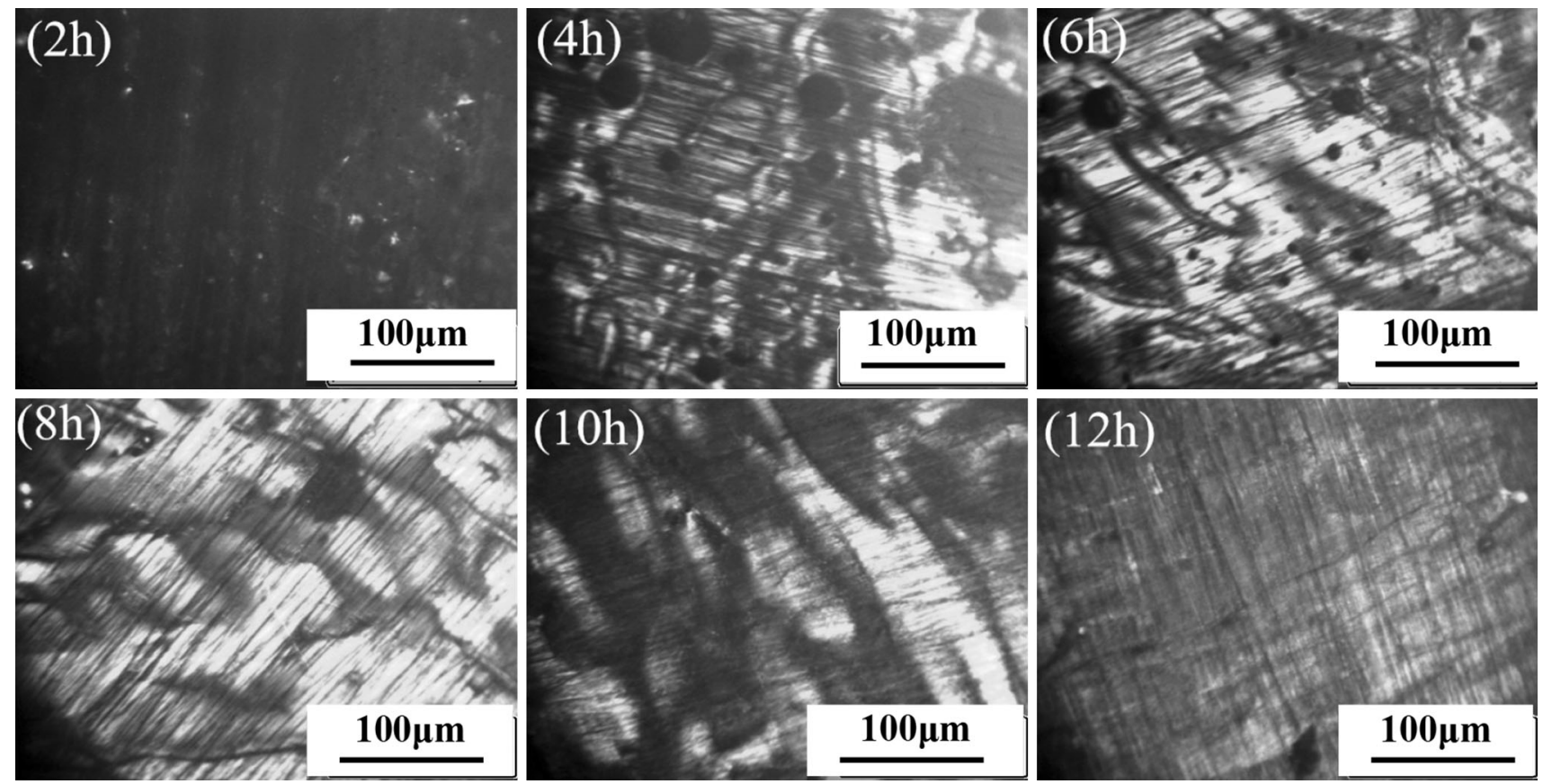

Fig. 6 Optical micrographs of R2-MP
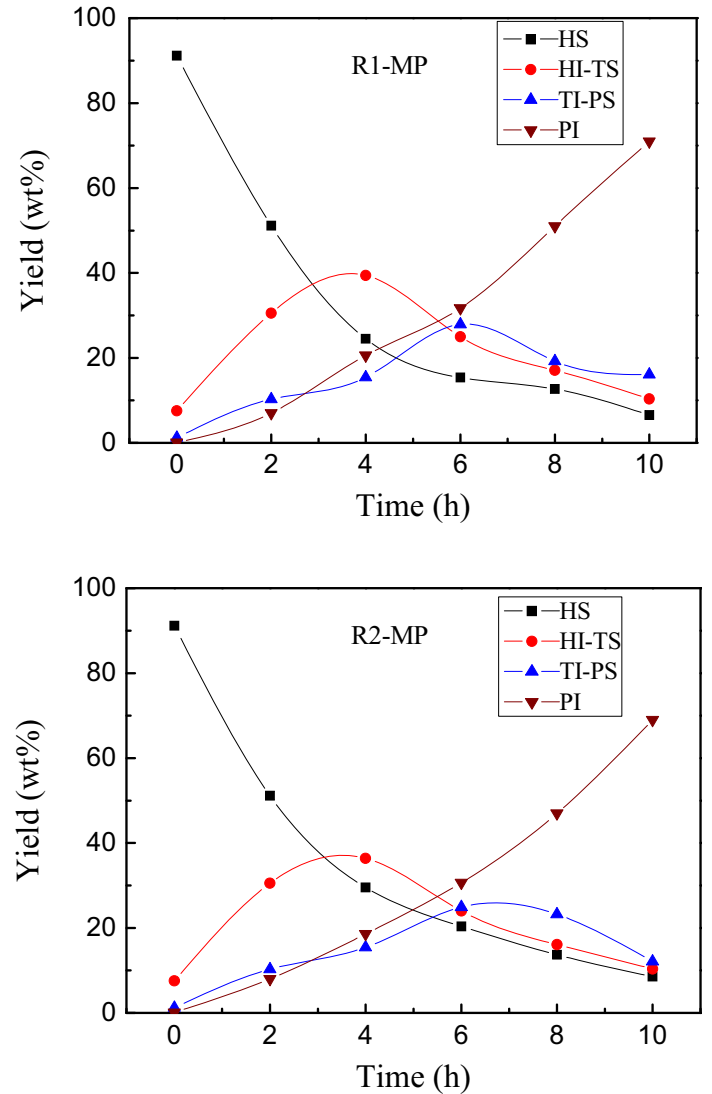

Fig. 7 Variation of HS, HI-TS, TI-PS and PI yields (wt\%) with time

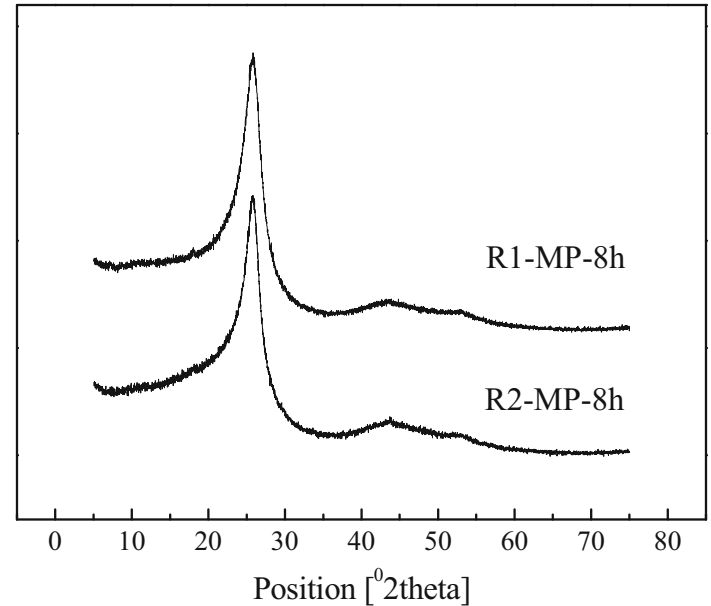

Fig. 8 XRD of the mesophase pitches

radicals to restrain the thermal polycondensation reaction. Thereby the system viscosity increased slowly. The lower system viscosity could help fulfill the transformation of each component, so the intermediate components were converted into the larger molecular components rapidly once they were generated. It was beneficial to generate the mesophase pitch with homogeneous molecular structure (molecular weight distribution was more concentrated). 
Table 4 X-ray parameters of the mesophase pitches

\begin{tabular}{lllll}
\hline Code & $29 /^{\circ}$ & $d_{002}(\mathrm{~nm})$ & $L_{\mathrm{c}}(\mathrm{nm})$ & $O_{\mathrm{g}}$ \\
\hline R1-MP-8h & 25.6686 & 0.3314 & 2.1 & 0.7425 \\
R2-MP-8h & 25.7437 & 0.3479 & 3.9 & 0.9713 \\
\hline
\end{tabular}

\section{XRD analysis}

The solubility of mesophase molecules in an isotropic matrix was determined by the distribution of mesophase molecule structures, and the movement of mesophase molecules was determined by the solubility of mesophase molecules. Thereby it affected the orientation of the aromatic layer in mesophase pitch. The X-ray spectra of R1MP-8h and R2-MP-8h are shown in Fig. 8 and the X-ray dates are tabulated in Table 4. Two kinds of mesophase pitches exhibited strong diffraction peaks as shown in Fig. 8, which indicated that the mesophase pitches were highly crystallized. Compared with R1-MP-8h, the stack height $\left(L_{\mathrm{c}}\right)$ of R2-MP-8h was higher, the interlayer spacing $\left(d_{002}\right)$ was lager and alignment degree $\left(O_{\mathrm{g}}\right)$ was improved. As a result, the crystal structure of R2-MP-8h was more ordered.

Compared with R2, R1 contains more alkyl side chains, which would produce more free radicals to increase the viscosity rapidly through condensation. Then the anisotropic structure is formed when the size of molecule reaches certain level. On the other side, R2 contains more naphthenic groups. The large content of naphthenic groups leads to hydrogen transfer reaction which plays a role in easing the carbonizing reaction, so that the viscosity of the system is lowered and then the mesophase pitch molecules have enough time to rearrange. Therefore, the optical texture of R1-MP-8h presents medium domain structure, while the optical texture of R2-MP-8h was large-flow domains.

\section{Conclusion}

The composition of raw materials played an important role on mesophase pitch formation. The raw material R2 with higher aromaticity, more naphthenic groups and less alkyl side chain was easy to form a high-quality mesophase pitch with large-domain optical texture, high mesophase content and low softening point $\left(243{ }^{\circ} \mathrm{C}\right)$. During the process of thermal treatments, the intermediate fractions HI-TS and TI-PS of R2-MP changed more slowly than those of R1MP and the thermal polycondensation reaction was restrained. The PAH of R2-MP-8h was less than that of R1MP-8h, which was consistent with the change of aromatic indexes. Additionally, the crystal structure of R2-MP-8h was more ordered, the stack height $\left(L_{\mathrm{c}}\right)$ was higher, the interlayer spacing $\left(d_{002}\right)$ was larger and the alignment degree $\left(O_{\mathrm{g}}\right)$ was improved.

Acknowledgments The authors gratefully acknowledge financial support from the National Natural Science Foundation of China (21176259) and the Fundamental Research Funds for the Central Universities (15CX05009A).

Conflict of interest The authors declare no competing financial interest.

Open Access This article is distributed under the terms of the Creative Commons Attribution 4.0 International License (http:// creativecommons.org/licenses/by/4.0/), which permits unrestricted use, distribution, and reproduction in any medium, provided you give appropriate credit to the original author(s) and the source, provide a link to the Creative Commons license, and indicate if changes were made.

\section{References}

1. Tate K, Yoshida H, Yanagida K (1987) Pitch for production of carbon fibers. U.S. Patent 4,670,129

2. Fathollahi B, Chau PC, White JL (2005a) Injection and stabilization of mesophase pitch in the fabrication of carboncarbon composites. Part II. Stabilization process. Carbon 43(1):131-141

3. Fathollahi B, Chau PC, White JL (2005b) Injection and stabilization of mesophase pitch in the fabrication of carbon-carbon composites. Part I. Injection process. Carbon 43(1):125-133

4. Fathollahi B, Jones B, Chau PC, White JL (2005c) Injection and stabilization of mesophase pitch in the fabrication of carboncarbon composites. Part III. mesophase stabilization at low temperatures and elevated oxidation pressures. Carbon 43(1):143-151

5. Korai Y, Ishida S, Yoon SH, Mochida I, Nakagawa Y, Yamaguchi C, Matsumura Y, Sakai Y, Fortin F, Yoon SH, Korai Y, Mochida I (1994) Reorganization of molecular alignment in naphthalene and methylnaphthalene derived pitches. Carbon 32(5):979-989

6. Miyake M, Ida T, Yoshida H, Wakisaka S, Nomure M (1993) Effects of reductively introduced alkyl groups and hydrogen to mesophase pitch on carbonization properties. Carbon 31(5):705-714

7. Yang KS, Kim YA, An KH, Yoon SH, Son TW, Mochida I (1997) Modification of naphthalene-derived mesophase pitch with benzoquinone. Carbon 35(7):923-928

8. Shin S, Jang J, Yoon SH, Mochida I (1997) A study on the effect of heat treatment on functional groups of pitch based activated carbon fiber using FTIR. Carbon 35(12):1739-1743

9. Mochida I, Fei YQ, Korai Y, Oishi T (1990) Co-carbonization of ethylene tar pitch and coal tar pitch to form needle coke. Fuel 69(6):672-677

10. Mochida I, Shimizu K, Korai Y, Sakai Y, Fujiyama S, Toshima H, Hono T (1992) Mesophase pitch catalytically prepared from anthracene with $\mathrm{HF} / \mathrm{BF}_{3}$. Carbon 30(1):55-61

11. Mochida I, Korai Y (1989) Carbonization in the tube bomb leading to needle coke: I. Cocarbonization of a petroleum vacuum residue and a FCC-decant oil into better needle coke. Carbon 27(3):359-365

12. Korai Y, Mochida I (1992) Molecular assembly of mesophase and isotropic pitches at their fused states. Carbon 30(7):1019-1024

13. Qian SA, Xiao YX, Gu YD (1987) Study on chemical composition and formation mechanism of some typical pitch feedstocks using field desorption mass spectrometry. Fuel 66(2):242-249 
14. Lin X, Ideta K, Miyawaki J, Takebe H, Yoon SH, Mochida I (2012) Correlation between fluidity properties and local structures of three typical Asian coal ashes. Energy Fuels 26(4):2136-2144

15. Mochida I, Korai Y, Ku CH, Watanabe F, Sakai Y (2000) Chemistry of synthesis, structure, preparation and application of aromatic-derived mesophase pitch. Carbon 38(2):305-328

16. Zhang J, Guo S, Wang Y, Xie D, Wang Z (2003) Effect of electrolytic systems on electrochemical hydrogenation of mesophase coal tar pitch. Fuel Process Technol 80(1):81-90

17. Guillen MD, Iglesias MJ, Dominguez A, Blanco CG (1992) Semi-quantitative FTIR analysis of a coal tar pitch and its extracts and residues in several organic solvents. Energy Fuels 6(4):518-525

18. Liang WJ, Que GH, Liu CG, Yang QS (2009) Petroleum chemistry. ShanDong, China university of petroleum press 54-90:106-107

19. Alvarez P, Diez N, Blanco C, Santamaria R, Menendez R, Granda M (2013) An insight into the polymerization of anthracene oil to produce pitch using nuclear magnetic resonance. Fuel 105:471-476
20. Morgan TJ, Alvarez-Rodriguez P, George A, Herod AA, Kandiyoti R (2010) Characterization of Maya crude oil maltenes and asphaltenes in terms of structural parameters calculated from nuclear magnetic resonance (NMR) spectroscopy and laser desorption-mass spectroscopy (LD-MS). Energy Fuels 24(7):3977-3989

21. Morgan TJ, Kandiyoti R (2014) Pyrolysis of coals and biomass: analysis of thermal breakdown and its products. Chem Rev 114(3):1547-1607

22. Mochida I, Kudo K, Takeshita K, Takashi R, Furumi YJ (1974) Modifying carbonization properties of pitches. I. Conversion of benzene-insoluble matter of coal-tar pitch into graphitizable carbon. Fuel 53(4):253-257

23. Mochida I, Tomari Y, Maeda K, Takeshita K (1975) Modifying carbonization properties of pitches. II. Attempts to improve the yield of fusible material from the quinoline-insoluble fraction of a petroleum pitch. Fuel 54(4):265-268 